\title{
Statistical field theory for simple fluids: the collective variables representation
}

\author{
J.-M. Caillol ${ }^{\text {a }}$ \\ ${ }^{a}$ Laboratoire de Physique Théorique CNRS UMR 8627, Bât. 210, Université de \\ Paris-Sud, 91405 Orsay Cedex, France \\ O. Patsahan ${ }^{\text {b,* }}$, I. Mryglod ${ }^{\mathrm{b}}$ \\ ${ }^{\mathrm{b}}$ Institute for Condensed Matter Physics of the National Academy of Sciences of \\ Ukraine, 1 Svientsitskii Str., 79011 Lviv, Ukraine
}

\begin{abstract}
An alternative representation of an exact statistical field theory for simple fluids, based on the method of collective variables, is presented. The results obtained are examined from the point of another version of theory that was developed recently by performing a Hubbard-Stratonovich transformation of the configurational Boltzmann factor [J.-M. Caillol, Mol. Phys. 101 (2003) 1617]. The analytical expressions for the pressure and the free energy are derived in two-loop approximation for both versions of theory and it is shown that they are indeed equivalent. The results yield a new type approximation within an untested approximation scheme.
\end{abstract}

Key words: Statistical field theory, loop expansion, collective variables PACS: 05.20.-y, 05.70.Ce

\section{Introduction}

Functional methods in modern statistical physics represent one of the most powerful tools for the study both of equilibrium and dynamical properties (see, e.g. [1,2]). A great amount of statistical field theories known in the literature are based of the Hubbard-Stratonovich transformation [3,4], proposed in

\footnotetext{
* Corresponding author.

Email addresses: Jean-Michel.Caillol@th.u-psud.fr (J.-M. Caillol), oksana@icmp.Iviv.ua (O. Patsahan), mryglod@icmp.lviv.ua (I. Mryglod).
} 
the 50ies. Nearly at the same time another method - so-called collective variables (CV) method - that allows in a explicit way to construct a functional representation for many-particle interacting systems was developed $[5,6]$ and applied for the description of charged particle systems, in particular, to the calculation of the configurational integral of the Coulomb systems. The idea of this method was based on: (i) the concept of collective coordinates being appropriate for the physics of system considered (see, for instance, [7]), and (ii) the integral equality allowing to derive an exact functional representation for the configurational Boltzmann factor. Later the CV methods was successfully developed for the description of classical many-particle systems [8] and for the phase transition theory [9]. One more functional approach, the mesoscopic field theory, was recently developed for the study of phase transitions in ionic fluids [10].

One of the goals of this paper is to reconsider the CV method from the point of view of the statistical field theory and to compare the results obtained with those found recently by one of us by means of the KSSHE (Kac-SiegertStratonovich-Hubbard-Edwards) theory [11].

We formulate the method of CV in real space and consider a one-component continuous model consisting of hard spheres interacting through additive pair potentials. The expression for the functional of the grand partition function is derived and the CV action that depends upon two scalar fields - field $\rho$ connected to the number density of particles and field $\omega$ conjugate to $\rho$ - is calculated. We study correlations between these fields as well as their relations to the density and energy correlations of the fluid. The grand partition function of the model is evaluated in a systematic way using a well-known method of statistical field theory, namely the so-called loop expansion. It consists in expanding functionally the action $\mathcal{H}$ around a saddle point, so that the lowest order (zero loop) approximation defines the mean-field (MF) level of the theory and the first order loop expressions correspond to the random phase approximation (RPA). Recently [11] this technique was applied to the action obtained within the framework of the KSSHE theory. In this paper we perform a two-loop expansion of the pressure and the free energy of the homogeneous fluid which yields a new type of approximation which we plan to test in our future work.

The paper is organized as follows. In Section 2, starting from the Hamiltonian, we introduce the two different functional representations of the grand partition function based on the KSSHE and CV methods. Here we also enter several types of statistical field averages that are important in the further part of the paper. In Section 3 we introduce the CV and KSSHE field correlation functions, establish links between them as well as their relation to the density correlation functions of the fluid. The MF level of the KSSHE and CV field theories is formulated in Section 4. Section 5 is devoted to the loop expansion 
of the grand potential. The pressure and the free energy of the homogeneous fluid are obtained in the two-loop approximation in Section 6. We conclude with some final remarks in Section 7.

\section{Summary of previous works}

\subsection{The model}

We consider the case of a simple three dimensional fluid that consists of identical hard spheres of diameter $\sigma$ with additional isotropic pair interactions $v\left(r_{i j}\right)\left(r_{i j}=\left|\mathbf{x}_{i}-\mathbf{x}_{j}\right|, \mathbf{x}_{i}\right.$ is the position of particle " $\left.i "\right)$. Since $v(r)$ is arbitrary in the core, i.e. for $r \leq \sigma$, we assume that $v(r)$ has been regularized in such a way that its Fourier transform $v_{q}$ is a well behaved function of $q$ and that $v(0)$ is a finite quantity. We denote by $\Omega$ the domain of volume $V$ occupied by the molecules of the fluid. The fluid is at equilibrium in the grand canonical (GC) ensemble and we denote by $\beta=1 / k T$ the inverse temperature ( $k$ is the Boltzmann constant) and $\mu$ is the chemical potential. In addition the particles are subject to an external potential $\psi(\mathbf{x})$ and we will denote by $\nu(\mathbf{x})=\beta(\mu-\psi(\mathbf{x}))$ the dimensionless local chemical potential. We will stick to notations usually adopted in standard textbooks on the theory of liquids (see e.g. [12]) and denote by $w(r)=-\beta v(r)$ the negative of the dimensionless pair interaction. Quite arbitrarily we will say that the interaction is attractive if the Fourier transform $\widetilde{w}(q)$ is positive for all $q$; in the converse case it will be said repulsive.

In a given configuration $\mathcal{C}=\left(N ; \mathbf{x}_{1} \ldots \mathbf{x}_{N}\right)$ the microscopic density of particles reads

$$
\widehat{\rho}(\mathbf{x} \mid \mathcal{C})=\sum_{i=1}^{N} \delta^{(3)}\left(\mathbf{x}-\mathbf{x}_{i}\right)
$$

and the GC partition function $\Xi[\nu]$ can thus be written as

$$
\Xi[\nu]=\sum_{N=0}^{\infty} \frac{1}{N !} \int_{\Omega} d 1 \ldots d n \exp \left(-\beta V_{\mathrm{HS}}(\mathcal{C})+\frac{1}{2}\langle\widehat{\rho}|w| \hat{\rho}\rangle+\langle\bar{\nu} \mid \widehat{\rho}\rangle\right)
$$

where $i \equiv \mathbf{x}_{i}$ and $d i \equiv d^{3} x_{i}$. For a given volume $V, \Xi[\nu]$ is a function of $\beta$ and a convex functional of the local chemical potential $\nu(x)$ which we have strengthened by using a bracket. In eq. $(2) \exp \left(-\beta V_{\mathrm{HS}}(\mathcal{C})\right)$ denotes the hard sphere contribution to the Boltzmann factor in a configuration $\mathcal{C}$ and $\bar{\nu}=\nu+\nu_{S}$ where $\nu_{S}=-w(0) / 2$ is proportional to the self-energy of the particles. From our hypothesis on $w(r), \nu_{S}$ is a finite quantity which depends however on the regularization of the potential in the core. In the r.h.s of eq. (2) we have also introduced Dirac's brac-kets notations 


$$
\begin{aligned}
\langle\bar{\nu} \mid \widehat{\rho}\rangle & \equiv \int_{\Omega} d 1 \bar{\nu}(1) \widehat{\rho}(1) \\
\langle\widehat{\rho}|w| \widehat{\rho}\rangle & \equiv \int_{\Omega} d 1 d 2 \widehat{\rho}(1 \mid \mathcal{C}) w(1,2) \widehat{\rho}(2 \mid \mathcal{C}) .
\end{aligned}
$$

Previous work have shown that $\Xi[\nu]$ can be rewritten as a functional integral. Two such equivalent representations are reviewed below.

\subsection{The KSSHE representation}

As it is well known the GC partition function $\Xi[\nu]$ can be re-expressed as a functional integral by performing the KSSHE transformation $[13,14,4,3,15,16]$ of the Boltzmann's factor. Under this transformation $\Xi[\nu]$ can be rewritten as the GC partition function of a fluid of bare hard spheres in the presence of a random Gaussian field $\varphi$ with a covariance given by the pair potential $[17,19,11,18]$. More precisely one has

- i) in the attractive case $(\widetilde{w}(q)>0)$

$$
\begin{aligned}
\Xi[\nu] & =\mathcal{N}_{w}^{-1} \int \mathcal{D} \varphi \exp \left(-\frac{1}{2}\left\langle\varphi\left|w^{-1}\right| \varphi\right\rangle\right) \Xi_{\mathrm{HS}}[\bar{\nu}+\varphi] \\
& \equiv\left\langle\Xi_{\mathrm{HS}}[\bar{\nu}+\varphi]\right\rangle_{w}
\end{aligned}
$$

- ii) in the repulsive case $(\widetilde{w}(q)<0)$

$$
\begin{aligned}
\Xi[\nu] & =\mathcal{N}_{(-w)}^{-1} \int \mathcal{D} \varphi \exp \left(\frac{1}{2}\left\langle\varphi\left|w^{-1}\right| \varphi\right\rangle\right) \Xi_{\mathrm{HS}}[\bar{\nu}+i \varphi] \\
& \equiv\left\langle\Xi_{\mathrm{HS}}[\bar{\nu}+i \varphi]\right\rangle_{(-w)}
\end{aligned}
$$

where, in both cases, $\varphi$ is a real random field and $\Xi_{\mathrm{HS}}$ denotes the GC partition function of bare hard spheres. $\Xi$ can thus be written as a Gaussian average $\langle\ldots\rangle_{w}$ of covariance $w$ and we have noted by $\mathcal{N}_{w}$ the normalization constant

$$
\mathcal{N}_{w}=\int \mathcal{D} \varphi \exp \left(-\frac{1}{2}\left\langle\varphi\left|w^{-1}\right| \varphi\right\rangle\right)
$$

The functional integrals which enter eqs. (4), (5) and (6) can be given a precise meaning in the case where the domain $\Omega$ is a cube of side $L$ with periodic boundary conditions (PBC) which will be implicitly assumed henceforth. More details are given in Appendix A.

In the repulsive case, the hard core part of the interaction is not compulsory for the existence of a thermodynamic limit [20] and the reference system can be chosen as the ideal gas $[21,22]$.

Eqs (4) and (5) are easily generalized to the case of molecular fluids or mixtures, for instance a charged hard spheres mixture (primitive model)[18,23]. 
When the pair interaction $w$ is neither attractive nor repulsive it is necessary to introduce two real scalar fields if some rigor is aimed at [11]. Alternatively, eq. (4) can be considered to hold in any case having in mind that $\varphi$ can be a complex scalar field. Therefore we shall write formally in all cases

$$
\Xi[\nu]=\mathcal{N}_{w}^{-1} \int \mathcal{D} \varphi \exp \left(-\mathcal{H}_{\mathrm{K}}[\nu, \varphi]\right)
$$

where the action of the KSSHE field theory reads as

$$
\mathcal{H}_{K}[\nu, \varphi]=\frac{1}{2}\left\langle\varphi\left|w^{-1}\right| \varphi\right\rangle-\ln \Xi_{\mathrm{HS}}[\bar{\nu}+\varphi]
$$

\subsection{The CV representation}

We introduce now briefly the CV representation of $\Xi[\nu]$ and refer the reader

to the literature for a more detailed presentation $[9,6,8,24,25]$. The starting point is the identity

$$
\begin{aligned}
\exp \left(\frac{1}{2}\langle\widehat{\rho}|w| \hat{\rho}\rangle\right) & =\int \mathcal{D} \rho \delta_{\mathcal{F}}[\rho-\hat{\rho}] \exp \left(\frac{1}{2}\langle\rho|w| \rho\rangle\right) \\
& =\int \mathcal{D} \rho \mathcal{D} \omega \exp \left(\frac{1}{2}\langle\rho|w| \rho\rangle+i\langle\omega \mid\{\rho-\hat{\rho}\}\rangle\right),
\end{aligned}
$$

where we have made use of the functional "delta function" $[1] \delta_{\mathcal{F}}[\rho]$ defined in eq. (A.6) in appendix A. Inserting eq. (9) in the expression (2) of the GC partition function one finds

$$
\Xi[\nu]=\int \mathcal{D} \rho \mathcal{D} \omega \exp \left(-\mathcal{H}_{\mathrm{CV}}[\nu, \rho, \omega]\right)
$$

where the action of the CV field theory reads as

$$
\mathcal{H}_{\mathrm{CV}}[\nu, \rho, \omega]=-\frac{1}{2}\langle\rho|w| \rho\rangle-i\langle\omega \mid \rho\rangle-\ln \Xi_{\mathrm{HS}}[\bar{\nu}-i \omega]
$$

We stress that $\omega$ and $\rho$ are two real scalar fields and that eqs. (10) and (11) are valid for repulsive, attractive as well as arbitrary pair interactions. Moreover, with the clever normalization of Wegner [19] for the functional measures there are no unspecified multiplicative constant involved in eq. (10) (see Appendix A for more details).

The CV transformation is clearly more general than the KSSHE transformation since it can be used for a pair interaction $w(1,2)$ which does not possess an inverse and is easily generalized for n-body interactions with $n>2$. The equivalence of the $\mathrm{CV}$ and KSSHE representations (7) and (10) of $\Xi[\nu]$ is 
readily established in the repulsive case $(w<0)$ by making use of the properties of Gaussian integrals (cf. eq. (A.5) of Appendix A). In the attractive or in the general case we did not find a convincing way to obtain one formula from the other.

\subsection{Statistical average}

In the sequel it will be important to distinguish carefully, besides the usual GC average $\left\langle\mathcal{A}(\mathcal{C})>_{\mathrm{GC}}\right.$ of a dynamic variable $\mathcal{A}(\mathcal{C})$, between several types of statistical field averages. Firstly Gaussian averages of covariance $w$

$$
\langle\mathcal{A}[\varphi]\rangle_{w}=\mathcal{N}_{w}^{-1} \int \mathcal{D} \varphi \exp \left(-\frac{1}{2}\left\langle\varphi\left|w^{-1}\right| \varphi\right\rangle\right) \mathcal{A}[\varphi]
$$

where $\mathcal{A}[\varphi]$ is some functional of the KSSHE field $\varphi$ and $\mathcal{N}_{w}$ has been defined in eq. (6); secondly the KSSHE averages defined as

$$
\langle\mathcal{A}[\varphi]\rangle_{\mathrm{K}}=\Xi[\nu]^{-1} \int \mathcal{D} \varphi \mathcal{A}[\varphi] \exp \left(-\mathcal{H}_{\mathrm{K}}[\nu, \varphi]\right)
$$

and thirdly the CV averages defined in a similar way as

$$
\langle\mathcal{A}[\rho, \omega]\rangle_{\mathrm{CV}}=\Xi[\nu]^{-1} \int \mathcal{D} \rho \mathcal{D} \omega \mathcal{A}[\rho, \omega] \exp \left(-\mathcal{H}_{\mathrm{CV}}[\nu, \rho, \omega]\right)
$$

where $\mathcal{A}[\rho, \omega]$ is a functional of the two $\mathrm{CV}$ fields $\rho$ an $\omega$.

\section{Correlation functions}

Since all thermodynamic quantities of the fluid can be expressed in terms of the GC density correlation functions $G^{(n)}[12]$ it is important to relate them to the field correlation functions in the CV and the KSSHE representations; this is subject of section 3 .

\subsection{Density correlations}

The ordinary and truncated (or connected) density correlation functions of the fluid will be defined in this paper as $[12,26,27]$ 


$$
\begin{aligned}
G^{(n)}[\nu](1, \ldots, n) & =\left\langle\prod_{1=1}^{n} \hat{\rho}\left(\mathbf{x}_{i} \mid \mathcal{C}\right)\right\rangle_{G C}=\frac{1}{\Xi[\nu]} \frac{\delta^{n} \Xi[\nu]}{\delta \nu(1) \ldots \delta \nu(n)}, \\
G^{(n), T}[\nu](1, \ldots, n) & =\frac{\delta^{n} \log \Xi[\nu]}{\delta \nu(1) \ldots \delta \nu(n)} .
\end{aligned}
$$

Our notation emphasizes the fact that the $G^{(n)}$ (connected and not connected) are functionals of the local chemical potential $\nu(x)$ and functions of the coordinates $(1, \ldots, n) \equiv\left(\mathbf{x}_{1}, \ldots, \mathbf{x}_{n}\right)$.

We know from the theory of liquids that $[26,27]$

$$
G^{(n), T}[\nu](1, \ldots, n)=G^{(n)}[\nu](1, \ldots, n)-\sum \prod_{m<n} G^{(m), T}[\nu]\left(i_{1}, \ldots, i_{m}\right),
$$

where the sum of products is carried out over all possible partitions of the set $(1, \ldots, n)$ into subsets of cardinal $m<n$. Of course $\rho[\nu](x) \equiv G^{(n=1)}[\nu](x)=$ $G^{(n=1), T}[\nu](x)$ is the local density of the fluid.

It follows from the definition $(15)$ of the $G^{(n)}[\nu](1, \ldots, n)$ that they can be reexpressed as KSSHE or CV statistical averages, i.e.

$$
\begin{aligned}
& G^{(n)}[\nu](1, \ldots, n)=\left\langle G_{\mathrm{HS}}^{(n)}[\bar{\nu}+\varphi](1, \ldots, n)\right\rangle_{\mathrm{K}} \\
& G^{(n)}[\nu](1, \ldots, n)=\left\langle G_{\mathrm{HS}}^{(n)}[\bar{\nu}-i \omega](1, \ldots, n)\right\rangle_{\mathrm{CV}}
\end{aligned}
$$

Although enlightening these relations are not very useful except for the special case $n=1$ which reads explicitly as

$$
\begin{aligned}
& \rho[\nu](\mathbf{x})=\left\langle\rho_{\mathrm{HS}}[\bar{\nu}+\varphi](\mathbf{x})\right\rangle_{\mathrm{K}}, \\
& \rho[\nu](\mathbf{x})=\left\langle\rho_{\mathrm{HS}}[\bar{\nu}-i \omega](\mathbf{x})\right\rangle_{\mathrm{CV}}
\end{aligned}
$$

where $\rho_{\mathrm{HS}}[\xi](\mathbf{x})$ is the local density of the hard sphere fluid with the local chemical potential $\xi(\mathbf{x})$.

\subsection{KSSHE field correlations}

Let us introduce the modified partition function

$$
\Xi^{1}[\nu, J]=\mathcal{N}_{w}^{-1} \int \mathcal{D} \varphi \exp \left(-\mathcal{H}_{\mathrm{K}}[\nu, \varphi]+\langle J \mid \varphi\rangle\right)
$$

where $J$ is a real scalar field. Clearly $\Xi^{1}[\nu, J]$ is the generator of field correlation functions and a standard result of statistical field theory yields [2] 


$$
\begin{aligned}
G_{\varphi}^{(n)}[\nu](1, \ldots, n) & =\left\langle\prod_{i=1}^{n} \varphi\left(\mathbf{x}_{i}\right)\right\rangle_{\mathrm{K}}=\left.\frac{1}{\Xi[\nu]} \frac{\delta^{n} \Xi^{1}[\nu, J]}{\delta J(1) \ldots \delta J(n)}\right|_{J=0}, \\
G_{\varphi}^{(n), T}[\nu](1, \ldots, n) & =\left.\frac{\delta^{n} \log \Xi^{1}[\nu, J]}{\delta J(1) \ldots \delta J(n)}\right|_{J=0} .
\end{aligned}
$$

Moreover one has [2]

$$
G_{\varphi}^{(n), T}(1, \ldots, n)=G_{\varphi}^{(n)}(1, \ldots, n)-\sum \prod_{m<n} G_{\varphi}^{(m), T}\left(i_{1}, \ldots, i_{m}\right)
$$

The relations between the $G_{\varphi}^{(n), T}$ and the truncated density correlation functions $G^{(n), T}$ have been established elsewhere by one of us $[11,18,23]$. We summarize them below for future reference.

$$
\begin{aligned}
\rho[\nu](1) & =w^{-1}\left(1,1^{\prime}\right)\left\langle\varphi\left(1^{\prime}\right)\right\rangle_{\mathrm{K}}, \\
G^{(2), T}[\nu](1,2) & =-w^{-1}(1,2)+w^{-1}\left(1,1^{\prime}\right) w^{-1}\left(2,2^{\prime}\right) \times \\
& \times G_{\varphi}^{(2), T}[\nu]\left(1^{\prime}, 2^{\prime}\right), \\
G^{(n), T}[\nu](1, \ldots, n) & =w^{-1}\left(1,1^{\prime}\right) \ldots w^{-1}\left(n, n^{\prime}\right) \times \\
& \times G_{\varphi}^{(n), T}[\nu]\left(1^{\prime}, \ldots, n^{\prime}\right) \quad \text { for } n \geq 3,
\end{aligned}
$$

where we have adopted Einstein's convention, i.e. a space integration of position variables labled by the same dummy indices over the domain $\Omega$ is meant.

\subsection{CV field correlations}

In this section we study the correlation functions of the fields $\rho$ and $\omega$ in the CV representation. We thus define

$$
\begin{aligned}
& G_{\rho}^{(n)}[\nu](1, \ldots, n)=\left\langle\prod_{1=1}^{n} \rho\left(\mathbf{x}_{i}\right)\right\rangle_{\mathrm{CV}}, \\
& G_{\omega}^{(n)}[\nu](1, \ldots, n)=\left\langle\prod_{1=1}^{n} \omega\left(\mathbf{x}_{i}\right)\right\rangle_{\mathrm{CV}},
\end{aligned}
$$

and their connected parts

$$
\begin{aligned}
& G_{\rho}^{(n), T}(1, \ldots, n)=G_{\rho}^{(n)}(1, \ldots, n)-\sum \prod_{m<n} G_{\rho}^{(m), T}\left(i_{1}, \ldots, i_{m}\right), \\
& G_{\omega}^{(n), T}(1, \ldots, n)=G_{\omega}^{(n)}(1, \ldots, n)-\sum \prod_{m<n} G_{\omega}^{(m), T}\left(i_{1}, \ldots, i_{m}\right) .
\end{aligned}
$$




\subsubsection{Correlation functions $G_{\rho}^{(n)}$}

Let us define the modified GC partition function

$$
\Xi^{2}[\nu, J]=\int \mathcal{D} \rho \mathcal{D} \omega \exp \left(-\mathcal{H}_{\mathrm{CV}}[\nu, \rho, \omega]+\langle J \mid \rho\rangle\right)
$$

where $J$ is a real scalar field. $\Xi^{2}[\nu, J]$ is clearly the generator of the $G_{\rho}^{(n)}$ and we thus have

$$
\begin{aligned}
G_{\rho}^{(n)}[\nu](1, \ldots, n) & =\left.\frac{1}{\Xi[\nu]} \frac{\delta^{n} \Xi^{2}[\nu, J]}{\delta J(1) \ldots \delta J(n)}\right|_{J=0}, \\
G_{\rho}^{(n), T}[\nu](1, \ldots, n) & =\left.\frac{\delta^{n} \log \Xi^{2}[\nu, J]}{\delta J(1) \ldots \delta J(n)}\right|_{J=0}
\end{aligned}
$$

The simplest way to obtain the relations between the $G_{\rho}^{(n)}$ and the density correlation functions is to start from the definition $(15)$ of $G^{(n)}$. One has

$$
\begin{aligned}
G^{(n)}(1, \ldots, n) & =\frac{1}{\Xi[\nu]} \frac{\delta^{n} \Xi[\nu]}{\delta \nu(1) \ldots \delta \nu(n)} \\
& =\frac{1}{\Xi[\nu]} \int \mathcal{D} \rho \mathcal{D} \omega \exp \left(\frac{1}{2}\langle\rho|w| \rho\rangle+i\langle\omega \mid \rho\rangle\right) \frac{\delta^{n} \Xi_{\mathrm{HS}}[\bar{\nu}-i \omega]}{\delta \nu(1) \ldots \delta \nu(n)} \\
& =\frac{1}{\Xi[\nu]} \int \mathcal{D} \rho \mathcal{D} \omega \exp \left(\frac{1}{2}\langle\rho|w| \rho\rangle+i\langle\omega \mid \rho\rangle\right) \times \\
& \times(i)^{n} \frac{\delta^{n} \Xi_{\mathrm{HS}}[\bar{\nu}-i \omega]}{\delta \omega(1) \ldots \delta \omega(n)} .
\end{aligned}
$$

Performing now $n$ integral by parts yields

$$
\begin{aligned}
G^{(n)}(1, \ldots, n) & =\frac{(-i)^{n}}{\Xi[\nu]} \int \mathcal{D} \rho \mathcal{D} \omega \exp \left(\frac{1}{2}\langle\rho|w| \rho\rangle+\ln \Xi_{\mathrm{HS}}[\bar{\nu}-i \omega]\right) \times \\
& \times \frac{\delta^{n} \exp (i\langle\omega \mid \rho\rangle)}{\delta \omega(1) \ldots \delta \omega(n)}=\left\langle\prod_{1=1}^{n} \rho(i)\right\rangle_{\mathrm{CV}}
\end{aligned}
$$

We have thus proved the expected result

$$
G^{(n)}[\nu](1, \ldots, n)=G_{\rho}^{(n)}[\nu](1, \ldots, n)
$$

which implies in turn that

$$
G^{(n), T}[\nu](1, \ldots, n)=G_{\rho}^{(n), T}[\nu](1, \ldots, n)
$$




\subsubsection{Correlation functions $G_{\omega}^{(n)}$}

Let us define the modified GC partition function

$$
\Xi^{3}[\nu, J]=\int \mathcal{D} \rho \mathcal{D} \omega \exp \left(-\mathcal{H}_{\mathrm{CV}}[\nu, \rho, \omega]+\langle J \mid \omega\rangle\right)
$$

where $J$ is a real scalar field. $\Xi^{3}[\nu, J]$ is the generator of the $G_{\omega}^{(n)}$ and we thus have

$$
\begin{aligned}
G_{\omega}^{(n)}[\nu](1, \ldots, n) & =\left.\frac{1}{\Xi[\nu]} \frac{\delta^{n} \Xi^{3}[\nu, J]}{\delta J(1) \ldots \delta J(n)}\right|_{J=0}, \\
G_{\omega}^{(n), T}[\nu](1, \ldots, n) & =\left.\frac{\delta^{n} \log \Xi^{3}[\nu, J]}{\delta J(1) \ldots \delta J(n)}\right|_{J=0}
\end{aligned}
$$

In order to relate $G_{\omega}^{(n)}$ to $G^{(n)}$ we perform the change of variable $\rho \rightarrow \rho+i J$ in eq. (29). The functional Jacobian of the transformation is of course equal to unity and one obtains the relation

$$
\ln \Xi^{3}[\nu, J]=-\frac{1}{2}\langle J|w| J\rangle+\ln \Xi^{2}[\nu, i w \star J]
$$

where the star $\star$ means a space convolution and $\Xi^{2}$ was defined at eq. (25). The idea is to perform now $n$ successive functional derivatives of both sides of eq. (31) with respect to $J$. Since it follows from the expression (26) of $G_{\rho}^{(n), T}$ that

$$
\begin{aligned}
\left.\frac{\delta^{n} \log \Xi^{2}[\nu, i w \star J]}{\delta J(1) \ldots \delta J(n)}\right|_{J=0} & =i^{n} w\left(1,1^{\prime}\right) \ldots w\left(n, n^{\prime}\right) \times \\
& \times G_{\rho}^{(n), T}[\nu]\left(1^{\prime}, \ldots, n^{\prime}\right),
\end{aligned}
$$

one readily obtains

$$
\begin{aligned}
\langle\omega(1)\rangle_{\mathrm{CV}} & =i w\left(1,1^{\prime}\right)\left\langle\rho\left(1^{\prime}\right)\right\rangle_{\mathrm{CV}}, \\
G_{\omega}^{(2), T}[\nu](1,2) & =-w(1,2) \\
& -w\left(1,1^{\prime}\right) w\left(2,2^{\prime}\right) G_{\rho}^{(2), T}[\nu]\left(1^{\prime}, 2^{\prime}\right), \\
G_{\omega}^{(n), T}[\nu](1, \ldots, n) & =i^{n} w\left(1,1^{\prime}\right) \ldots w\left(n, n^{\prime}\right) \times \\
& \times G_{\rho}^{(n), T}[\nu]\left(1^{\prime}, \ldots, n^{\prime}\right) \quad \text { for } n \geq 3 .
\end{aligned}
$$

A comparison between eqs (22), (28), and (33) will show that the CV and KSSHE correlation functions are simply related since we have, as expected

$$
G_{\varphi}^{(n), T}[\nu](1, \ldots, n)=(-i)^{n} G_{\omega}^{(n), T}[\nu](1, \ldots, n) \quad \text { for all } n
$$




\section{Mean Field theory}

\subsection{KSSHE representation}

We summarize previous work on the mean-field (MF) or saddle point approximation of the KSSHE theory $[11,18]$. At the MF level one has [2]

$$
\Xi_{\mathrm{MF}}[\nu]=\exp \left(-\mathcal{H}_{\mathrm{K}}\left[\nu, \varphi_{0}\right]\right)
$$

where, for $\varphi=\varphi_{0}$, the action is stationary, i.e.

$$
\left.\frac{\delta \mathcal{H}_{\mathrm{K}}[\nu, \varphi]}{\delta \varphi}\right|_{\varphi_{0}}=0
$$

Replacing the KSSHE action by its expression (8) in eq. (36) leads to an implicit equation for $\varphi_{0}$ :

$$
\varphi_{0}(1)=w\left(1,1^{\prime}\right) \rho_{\mathrm{HS}}\left[\bar{\nu}+\varphi_{0}\right]\left(1^{\prime}\right)
$$

which reduces to

$$
\varphi_{0}=\widetilde{w}(0) \rho_{\mathrm{HS}}\left[\bar{\nu}+\varphi_{0}\right]
$$

for a homogeneous system. It follows from the stationary condition (36) that the MF density is given by

$$
\rho_{\mathrm{MF}}[\nu](1)=\frac{\delta \ln \Xi_{\mathrm{MF}}[\nu]}{\delta \nu(1)}=\rho_{\mathrm{HS}}\left[\bar{\nu}+\varphi_{0}\right](1),
$$

and that the MF grand potential reads

$$
\ln \Xi_{\mathrm{MF}}[\nu]=\ln \Xi_{\mathrm{HS}}\left[\bar{\nu}+\varphi_{0}\right]-\frac{1}{2}\left\langle\rho_{\mathrm{MF}}|w| \rho_{\mathrm{MF}}\right\rangle
$$

Moreover, the MF Kohn-Scham free energy defined as the Legendre transform

$$
\beta \mathcal{A}_{\mathrm{MF}}[\rho]=\sup _{\nu}\left\{\langle\rho \mid \nu\rangle-\ln \Xi_{\mathrm{MF}}[\nu]\right\}
$$

is found to be

$$
\beta \mathcal{A}_{\mathrm{MF}}[\rho]=\beta \mathcal{A}_{\mathrm{HS}}[\rho]-\frac{1}{2}\langle\rho|w| \rho\rangle+\frac{1}{2} \int_{\Omega} d x w(0) \rho(x) .
$$

It can be shown [11] that $\mathcal{A}_{\mathrm{MF}}[\rho]$ constitutes a rigorous upper bound for the exact free energy $\mathcal{A}[\rho]$ if the interaction is attractive $(\widetilde{w}(q)>0)$ and a lower bound in the converse case $(\widetilde{w}(q)<0)$.

Finally, the pair correlation and vertex functions at the zero-loop order which are defined respectively as 


$$
\begin{aligned}
G_{\mathrm{MF}}^{(2), T}[\nu](1,2) & =\frac{\delta^{2} \ln \Xi_{\mathrm{MF}}[\nu]}{\delta \nu(1) \delta \nu(2)}, \\
C_{\mathrm{MF}}^{(2)}[\rho](1,2) & =-\frac{\delta^{2} \beta \mathcal{A}_{\mathrm{MF}}[\rho]}{\delta \rho(1) \delta \rho(2)},
\end{aligned}
$$

are given by

$$
\begin{aligned}
G_{\mathrm{MF}}^{(2), T}(1,2) & =\left(1-w \cdot G_{\mathrm{HS}}^{(2), T}\left[\bar{\nu}+\varphi_{0}\right]\right)^{-1} \cdot G_{\mathrm{HS}}^{(2), T}\left[\bar{\nu}+\varphi_{0}\right](1,2) \\
C_{\mathrm{MF}}^{(2)}(1,2) & =-G_{\mathrm{MF}}^{(2), T-1}(1,2)=C_{\mathrm{HS}}(1,2)+w(1,2) .
\end{aligned}
$$

It follows then from eq. (22b) that we have

$$
G_{\varphi, \mathrm{MF}}^{(2), T}(1,2)=\left(1-w \cdot G_{\mathrm{HS}}^{(2)}\left[\bar{\nu}+\varphi_{0}\right]\right)^{-1} \cdot w(1,2)
$$

\subsection{CV representation}

An analysis similar to that of Sec 4.1 can be made in the CV representation. The MF level of the CV field theory will be defined by

$$
\Xi_{\mathrm{MF}}[\nu]=\exp \left(-\mathcal{H}_{\mathrm{CV}}\left[\nu, \rho_{0}, \omega_{0}\right]\right)
$$

where, for $\rho=\rho_{0}$ and $\omega=\omega_{0}$ the $\mathrm{CV}$ action is stationary, i.e.

$$
\left.\frac{\delta \mathcal{H}_{\mathrm{CV}}[\nu, \rho, \omega]}{\delta \rho}\right|_{\left(\rho_{0}, \omega_{0}\right)}=\left.\frac{\delta \mathcal{H}_{\mathrm{CV}}[\nu, \rho, \omega]}{\delta \omega}\right|_{\left(\rho_{0}, \omega_{0}\right)}=0 .
$$

Replacing the CV action by its expression (11) in eq. (47) yields a set of two coupled implicit equations for $\rho_{0}$ and $\omega_{0}$ :

$$
\begin{aligned}
& 0=w(1,2) \rho_{0}(2)+i \omega_{0}(1) \\
& 0=\rho_{0}(1)-\rho_{\mathrm{HS}}\left[\bar{\nu}-i \omega_{0}\right](1) .
\end{aligned}
$$

If we define $\varphi_{0}=-i \omega_{0}$, then the two previous equations can be rewritten

$$
\begin{aligned}
\varphi_{0} & =w(1,2) \rho_{0}(2), \\
\rho_{0}(1) & =\rho_{\mathrm{HS}}\left[\bar{\nu}+\varphi_{0}\right](1),
\end{aligned}
$$

which shows that, as expected, $\varphi_{0}$ coincides with the saddle point of the KSSHE field theory (cf Sec 4.1). Moreover a direct calculation will show that

$$
\ln \Xi_{\mathrm{MF}}[\nu]=-\mathcal{H}_{\mathrm{K}}\left[\nu, \varphi_{0}\right]=-\mathcal{H}_{\mathrm{CV}}\left[\nu, \rho_{0}, \omega_{0}\right]
$$


Therefore the local density, the grand potential, the free energy, the correlation and vertex functions coincide at the MF level in the CV and KSSHE field theories.

\section{Loop expansion of the grand potential}

A one loop expression for the grand potential $\ln \Xi[\nu]$ and the free energy $\beta \mathcal{A}[\rho]$ has been obtained in [11] in the framework of KSSHE field theory. Here we perform a two-loop expansion for $\ln \Xi$. The derivation will be made both in the KSSHE and CV representations.

\subsection{KSSHE representation}

The loop expansion of the logarithm of the partition function of a scalar field theory can be found in any standard text book, see e.g. that of Zinn-Justin [2]. One proceeds as follows. A small dimensionless parameter $\lambda$ is introduced and the loop expansion is obtained by the set of transformations

$$
\begin{aligned}
\varphi & =\varphi_{0}+\lambda^{1 / 2} \chi \\
\ln \Xi[\nu] & =\lambda \ln \left\{\mathcal{N}_{w}^{-1} \int \mathcal{D} \chi \exp \left(-\frac{\mathcal{H}_{\mathrm{K}}[\nu, \varphi]}{\lambda}\right)\right\}, \\
& =\ln \Xi^{(0)}[\nu]+\lambda \ln \Xi^{(1)}[\nu]+\lambda^{2} \ln \Xi^{(2)}[\nu]+\mathcal{O}\left(\lambda^{3}\right),
\end{aligned}
$$

where $\varphi_{0}$ is the saddle point of the KSSHE action. At the end of the calculation one set $\lambda=1$. It follows from the stationary condition (36) that a functional Taylor expansion of $\mathcal{H}_{\mathrm{K}}[\nu, \varphi]$ about the saddle point has the form

$$
\begin{aligned}
\frac{\mathcal{H}_{\mathrm{K}}[\nu, \varphi]}{\lambda} & =\frac{\mathcal{H}_{\mathrm{K}}\left[\nu, \varphi_{0}\right]}{\lambda}+\frac{1}{2}\left\langle\chi\left|\Delta_{\varphi_{0}}^{-1}\right| \chi\right\rangle \\
& +\sum_{n=3}^{\infty} \frac{\lambda^{\frac{n}{2}-1}}{n !} \int_{\Omega} d 1 \ldots d n \mathcal{H}_{\varphi_{0}}^{(n)}(1, \ldots, n) \chi(1) \ldots \chi(n),
\end{aligned}
$$

where we have adopted Zinn-Justin's notations [2]. In eq. (52)

$$
\Delta_{\varphi_{0}}^{-1}(1,2)=w^{-1}(1,2)-G_{\mathrm{HS}}^{(2), T}\left[\bar{\nu}+\varphi_{0}\right](1,2) .
$$

$\Delta_{\varphi_{0}}(1,2)$ is the free propagator of the theory, it coincides with $G_{\varphi, \mathrm{MF}}^{(2)}(1,2)$ as can be seen by comparing eqs. (45) and (53). If $\nu$ is uniform then the system 
is homogeneous and $\Delta_{\varphi_{0}}(1,2)$ takes on a simple form in Fourier space, i.e.

$$
\widetilde{\Delta}_{\varphi_{0}}(q)=\frac{\widetilde{w}(q)}{1-\widetilde{w}(q) \widetilde{G}_{\mathrm{HS}}^{(2), T}\left[\bar{\nu}+\varphi_{0}\right](q)} .
$$

Finally the kernels $\mathcal{H}_{\varphi_{0}}^{(n)}$ in the r.h.s. of eq. (52) are given by

$$
\mathcal{H}_{\varphi_{0}}^{(n)}(1, \ldots, n) \equiv-G_{\mathrm{HS}}^{(n), T}\left[\bar{\nu}+\varphi_{0}\right](1, \ldots, n)
$$

The expression of $\ln \Xi^{(n)}[\nu]$ in terms of the propagator $\Delta_{\varphi_{0}}^{-1}(1,2)$ and the vertex interactions $\mathcal{H}_{\varphi_{0}}^{(n)}$ is obtained by means of a cumulant expansion of $\ln \Xi[\nu]$ and by making a repeated use of Wick's theorem.

Of course $\Xi^{(0)}[\nu] \equiv \Xi_{\mathrm{MF}}[\nu]$. At the one-loop order one finds [11]

$$
\Xi^{(1)}[\nu]=\frac{\mathcal{N}_{\Delta_{\varphi_{0}}}}{\mathcal{N}_{w}}=\frac{\int \mathcal{D} \varphi \exp \left(-\frac{1}{2}\left\langle\varphi\left|\Delta_{\varphi_{0}}\right| \varphi\right\rangle\right)}{\int \mathcal{D} \varphi \exp \left(-\frac{1}{2}\langle\varphi|w| \varphi\rangle\right)}
$$

For a homogeneous system the Gaussian integrals in the r.h.s. of eq. (56) can be performed explicitly (cf. eqs. (A.5) of Appendix A) and one has

$$
\ln \Xi^{(1)}[\nu]=-\frac{V}{2} \int_{\mathbf{q}} \ln \left(1-\widetilde{w}(q) \widetilde{G}_{\mathrm{HS}}^{(2), T}\left[\bar{\nu}+\varphi_{0}\right](q)\right)
$$

As is shown in detail in [11] the one-loop approximation coincides with the well known RPA approximation of the theory of liquids [12].

The second-loop order contribution $\ln \Xi^{(2)}$ to the grand potential has a complicated expression involving the sum of three diagrams sketched in fig. 1

$$
\ln \Xi^{(2)}[\nu]=D_{a}+D_{b}+D_{c}
$$

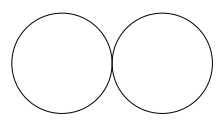

$\mathrm{D}_{\mathrm{a}}$

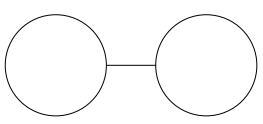

$\mathrm{D}_{\mathrm{b}}$

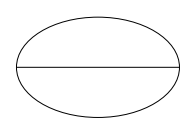

$\mathrm{D}_{\mathrm{c}}$

Fig. 1. Diagrams which contribute to $\ln \Xi^{(2)}[\nu] . D_{a}$ and $D_{c}$ are irreducible while $D_{b}$ is reducible.

More explicitly one has [2] 


$$
\begin{aligned}
D_{a} & =\frac{1}{8} \int_{\Omega} d 1 \ldots d 4 \Delta_{\varphi_{0}}(1,2) \Delta_{\varphi_{0}}(3,4) G_{\mathrm{HS}}^{(4), T}\left[\bar{\nu}+\varphi_{0}\right](1,2,3,4) \\
D_{b} & =\frac{1}{8} \int_{\Omega} d 1 \ldots d 3 d 1^{\prime} \ldots d 3^{\prime} \Delta_{\varphi_{0}}(1,2) \Delta_{\varphi_{0}}\left(1^{\prime}, 2^{\prime}\right) \Delta_{\varphi_{0}}\left(3,3^{\prime}\right) \\
& \times G_{\mathrm{HS}}^{(3), T}\left[\bar{\nu}+\varphi_{0}\right](1,2,3) G_{\mathrm{HS}}^{(3), T}\left[\bar{\nu}+\varphi_{0}\right]\left(1^{\prime}, 2^{\prime}, 3^{\prime}\right) \\
D_{c} & =\frac{1}{12} \int_{\Omega} d 1 \ldots d 3 d 1^{\prime} \ldots d 3^{\prime} \Delta_{\varphi_{0}}\left(1,1^{\prime}\right) \Delta_{\varphi_{0}}\left(2,2^{\prime}\right) \Delta_{\varphi_{0}}\left(3,3^{\prime}\right) \\
& \times G_{\mathrm{HS}}^{(3), T}\left[\bar{\nu}+\varphi_{0}\right](1,2,3) G_{\mathrm{HS}}^{(3), T}\left[\bar{\nu}+\varphi_{0}\right]\left(1^{\prime}, 2^{\prime}, 3^{\prime}\right)
\end{aligned}
$$

As they stand, the above relations are not particularly useful for practical applications (even in the homogeneous case) since they involve the three and four body correlation functions of the reference HS fluid. We will introduce some reasonable approximation in Sec. 6 to tackle with this horible expression. Quite remarkably it has been shown recently that for a symmetric mixture of charged hard spheres $\ln \Xi^{(2)}$ has a much more simple expression which involves only the pair correlation functions of the HS fluid as a consequence of local charge neutrality $[18,23]$.

\subsection{CV representation}

In order to obtain a loop expansion of $\ln \Xi$ in the $\mathrm{CV}$ representation we consider the following set of transformations

$$
\begin{aligned}
\rho & =\rho_{0}+\lambda^{1 / 2} \delta \rho, \\
\omega & =\omega_{0}+\lambda^{1 / 2} \delta \omega, \\
\ln \Xi[\nu] & =\lambda \ln \left\{\int \mathcal{D} \delta \rho \mathcal{D} \delta \omega \exp \left(-\frac{\mathcal{H}_{\mathrm{CV}}[\nu, \rho, \omega]}{\lambda}\right)\right\},
\end{aligned}
$$

where $\left(\rho_{0}, \omega_{0}\right)$ is the saddle point of the CV action. The form retained in eqs (60a) and (60b) is imposed by the exact relation $\langle\omega\rangle_{\mathrm{CV}}=i w \star\langle\rho\rangle_{\mathrm{CV}}$ derived in Sec 3.3.2. It follows from the stationary condition (47) that the

functional Taylor expansion of $\mathcal{H}_{\mathrm{CV}}[\nu, \rho, \omega]$ about the saddle point reads as

$$
\begin{aligned}
\frac{\mathcal{H}_{\mathrm{CV}}[\nu, \rho, \omega]}{\lambda} & =\frac{\mathcal{H}_{\mathrm{CV}}\left[\nu, \rho_{0}, \omega_{0}\right]}{\lambda}-\frac{1}{2}\langle\delta \rho|w| \delta \rho\rangle-i\langle\delta \omega \mid \delta \rho\rangle \\
& -\frac{1}{2}\left\langle\delta \omega\left|G_{\mathrm{HS}}^{(2), T}\left[\bar{\nu}-i \omega_{0}\right]\right| \delta \omega\right\rangle+\delta \mathcal{H}[\delta \omega]
\end{aligned}
$$

where

$$
\delta \mathcal{H}[\delta \omega]=-\sum_{n=3}^{\infty} \frac{(-i)^{n}}{n !} \lambda^{\frac{n}{2}-1} \int_{\Omega} d 1 \ldots d n \mathcal{H}_{\varphi_{0}}^{(n)}(1, \ldots, n) \delta \omega(1) \ldots \delta \omega(n),
$$


since, as $\varphi_{0}=-i \omega_{0}$ we have indeed $G_{\mathrm{HS}}^{(n), T}\left[\bar{\nu}-i \omega_{0}\right]=-\mathcal{H}_{\varphi_{0}}^{(n)}$ where the kernel $\mathcal{H}_{\varphi_{0}}^{(n)}$ is precisely that defined in eq. (55).

We are thus led to define a two-fields Gaussian Hamiltonian

$$
\mathcal{H}_{\mathrm{G}}[\rho, \omega] \equiv-\frac{1}{2}\langle\rho|w| \rho\rangle-i\langle\omega \mid \rho\rangle+\frac{1}{2}\left\langle\omega\left|G_{\mathrm{HS}}^{(2), T}\left[\bar{\nu}-i \omega_{0}\right]\right| \omega\right\rangle
$$

and Gaussian averages

$$
\langle\ldots\rangle_{\mathrm{G}} \equiv \mathcal{N}_{\mathrm{G}}^{-1} \int \mathcal{D} \rho \mathcal{D} \omega \ldots \exp \left(-\mathcal{H}_{\mathrm{G}}[\rho, \omega]\right)
$$

where the normalization constant $\mathcal{N}_{\mathrm{G}}$ is given by

$$
\mathcal{N}_{\mathrm{G}}=\int \mathcal{D} \rho \mathcal{D} \omega \exp \left(-\mathcal{H}_{\mathrm{G}}[\rho, \omega]\right)
$$

Note that if $w>0$ (attractive case ) eq. (64) makes sense only if the integration over the field variable $\omega$ is performed in first. With these notations in hands we can rewrite eq. (60c) as

$$
\ln \Xi[\nu]=\ln \Xi_{\mathrm{MF}}[\nu]+\lambda \ln \mathcal{N}_{\mathrm{G}}+\lambda \ln \left\langle e^{\delta \mathcal{H}[\omega]}\right\rangle_{\mathrm{G}} .
$$

A cumulant expansion of the last term in the r.h.s. yields the $\lambda$ expansion

$$
\ln \Xi=\ln \Xi_{\mathrm{MF}}+\lambda \ln \bar{\Xi}^{(1)}+\lambda\left\{\langle\delta \mathcal{H}\rangle_{\mathrm{G}}+\frac{1}{2}\left(\left\langle\delta \mathcal{H}^{2}\right\rangle_{\mathrm{G}}-\langle\delta \mathcal{H}\rangle_{\mathrm{G}}^{2}\right)\right\}+\mathcal{O}\left(\lambda^{3}\right)
$$

where

$$
\bar{\Xi}^{(1)}[\nu]=\mathcal{N}_{\mathrm{G}},
$$

and it will be shown that the third term in the r.h.s. of eq. (67) is of order $\mathcal{O}\left(\lambda^{2}\right)$.

\subsubsection{One-loop approximation}

We want to prove that the CV and KSSHE one-loop approximations for $\Xi$, respectively $\bar{\Xi}^{(1)}$ and $\Xi^{(1)}$, coincide. Let us first rewrite the formula (56) for $\Xi^{(1)}$ as the Gaussian average

$$
\Xi^{(1)}[\nu]=\left\langle\exp \left(\frac{1}{2}\left\langle\varphi\left|G_{\mathrm{HS}}^{(2), T}\right| \varphi\right\rangle\right)\right\rangle_{w}
$$

Now we focus on $\bar{\Xi}^{(1)}[\nu]=\mathcal{N}_{\mathrm{G}}$. Performing the (ordinary Gaussian) integration over $\omega$ first in eq. (65) we find 


$$
\begin{aligned}
\bar{\Xi}^{(1)}[\nu] & =\int \mathcal{D} \rho \exp \left(\frac{1}{2}\langle\rho|w| \rho\rangle\right) \int \mathcal{D} \omega \exp \left(i\langle\omega \mid \rho\rangle-\frac{1}{2}\left\langle\omega\left|G_{\mathrm{HS}}^{(2), T}\right| \omega\right\rangle\right) \\
& =\mathcal{N}_{\left[G_{\mathrm{HS}}^{(2), T}\right]^{-1}} \int \mathcal{D} \rho \exp \left(-\frac{1}{2}\left\langle\rho\left|\left[G_{\mathrm{HS}}^{(2), T}\right]^{-1}-w\right| \rho\right\rangle\right) .
\end{aligned}
$$

Now we make use of the two following properties of Gaussian integrals

$$
\begin{aligned}
\mathcal{N}_{a} & =1 / \mathcal{N}_{a^{-1}} \\
\left\langle\exp \left(\frac{1}{2}\langle\varphi|b| \varphi\rangle\right)\right\rangle_{a} & =\left\langle\exp \left(\frac{1}{2}\langle\varphi|a| \varphi\rangle\right)\right\rangle_{b}
\end{aligned}
$$

to rewrite

$$
\begin{aligned}
\bar{\Xi}^{(1)}[\nu] & =\frac{\int \mathcal{D} \rho \exp \left(-\frac{1}{2}\left\langle\rho\left|\left[G_{\mathrm{HS}}^{(2), T}\right]^{-1}-w\right| \rho\right\rangle\right)}{\int \mathcal{D} \rho \exp \left(-\frac{1}{2}\left\langle\rho\left|\left[G_{\mathrm{HS}}^{(2), T}\right]^{-1}\right| \rho\right\rangle\right)} \\
& =\left\langle\exp \left(\frac{1}{2}\langle\rho|w| \rho\rangle\right)\right\rangle_{G_{\mathrm{HS}}^{(2), T}}
\end{aligned}
$$

It follows then readily from eqs. (69) and (71b) that $\Xi^{(1)}=\Xi^{(1)}$. The two identities (71a) and (71b) are easy to show in the homogeneous case and, in this case, there are an immediate consequence of the properties of the Gaussian integral (A.5). In the general, non-homogeneous case, they are derived in Appendix B.

Let us first have a look at the correlations of the field $\rho$.

\subsubsection{Two-loop approximation}

At the two-loop level we have to compute the averages $\langle\delta \mathcal{H}[\omega]\rangle_{\mathrm{G}}$ and $\left\langle\delta \mathcal{H}[\omega]^{2}\right\rangle_{\mathrm{G}}$. In order to do so we have first to generalize Wick's theorem to this special kind of Gaussian average defined in eq. (64).

Let us first have a look at the correlations of the field $\rho$. One has from eq. (64)

$$
\begin{aligned}
\langle\rho(1) \ldots \rho(n)\rangle_{\mathrm{G}} & =\frac{\int \mathcal{D} \rho \mathcal{D} \omega \rho(1) \ldots \rho(n) \exp \left(-\mathcal{H}_{\mathrm{G}}[\rho, \omega]\right)}{\int \mathcal{D} \rho \mathcal{D} \omega \exp \left(-\mathcal{H}_{\mathrm{G}}[\rho, \omega]\right)} \\
& =\frac{\int \mathcal{D} \rho \rho(1) \ldots \rho(n) \exp \left(-\frac{1}{2}\left\langle\rho\left|\left[G_{\mathrm{HS}}^{(2), T}\right]^{-1}-w\right| \rho\right\rangle\right)}{\int \mathcal{D} \rho \exp \left(-\frac{1}{2}\left\langle\rho\left|\left[G_{\mathrm{HS}}^{(2), T}\right]^{-1}-w\right| \rho\right\rangle\right)},
\end{aligned}
$$


where we have performed the (ordinary) Gaussian integral on the field $\omega$ first. We are thus led to the ordinary Gaussian average (provided that $\left[G_{\mathrm{HS}}^{(2), T}\right]^{-1}-w$ is positive definite, which we assume), therefore the usual Wick's theorem is applied which yields

$$
\begin{aligned}
\langle\rho(1)\rangle_{\mathrm{G}}^{T} & =0 \\
\langle\rho(1) \rho(2)\rangle_{\mathrm{G}}^{T} & =\left(\left[G_{\mathrm{HS}}^{(2), T}\right]^{-1}-w\right)^{-1}=G_{\mathrm{MF}}^{(2), T}(1,2) \\
\langle\rho(1) \ldots \rho(n)\rangle_{\mathrm{G}}^{T} & =0(\text { for } n \geq 3) .
\end{aligned}
$$

We take now advantage of the general relations (33) between the truncated correlations of $\rho$ and $\omega$ in the $\mathrm{CV}$ formalism to get

$$
\begin{aligned}
\langle\omega(1)\rangle_{\mathrm{G}}^{T} & =0 \\
\langle\omega(1) \omega(2)\rangle_{\mathrm{G}}^{T} & =-w\left(1,1^{\prime}\right) w\left(2,2^{\prime}\right) G_{\mathrm{MF}}^{(2), T}\left(1^{\prime}, 2^{\prime}\right)=-\Delta_{\varphi_{0}}(1,2) \\
\langle\omega(1) \ldots \omega(n)\rangle_{\mathrm{G}}^{T} & =0(\text { for } n \geq 3),
\end{aligned}
$$

from which Wick's theorem follows

$$
\begin{aligned}
& \langle\omega(1) \ldots \omega(n)\rangle_{\mathrm{G}}=0(n \text { odd }), \\
& \langle\omega(1) \ldots \omega(n)\rangle_{\mathrm{G}}=(-1)^{n} \sum_{\text {pairs }} \prod \Delta_{\varphi_{0}}\left(i_{1}, i_{2}\right)(n \text { even }),
\end{aligned}
$$

which was an expected result thanks to the correspondence $\varphi_{K} \leftrightarrow i \omega_{C V}$. We have now at our disposal all the tools to compute

$$
\begin{aligned}
\langle\delta \mathcal{H}[\omega]\rangle_{\mathrm{G}} & =\frac{(-i)^{4}}{4 !} \lambda \int d 1 \ldots d 4 G_{\mathrm{HS}}^{(4)}\left[\bar{\nu}+\varphi_{0}\right](1, \ldots 4) \\
& \times\langle\omega(1) \ldots \omega(4)\rangle_{\mathrm{G}}+\mathcal{O}\left(\lambda^{2}\right) \\
& =\frac{\lambda}{8} \int d 1 \ldots d 4 G_{\mathrm{HS}}^{(4)}\left[\bar{\nu}+\varphi_{0}\right](1, \ldots 4) \\
& \times \Delta_{\varphi_{0}}(1,2) \Delta_{\varphi_{0}}(3,4)+\mathcal{O}\left(\lambda^{2}\right)
\end{aligned}
$$

where we have made use of Wick's theorem (75). We note that $\langle\delta \mathcal{H}[\omega]\rangle_{\mathrm{G}}^{2}$ will not contribute to $\ln \Xi$ at the two-loop order and it remains to compute $\left\langle\delta \mathcal{H}^{2}[\omega]\right\rangle_{\mathrm{G}}$. One finds 


$$
\begin{aligned}
\left\langle\delta \mathcal{H}^{2}[\omega]\right\rangle_{\mathrm{G}} & =\frac{\lambda}{(3 !)^{2}} \int d 1 \ldots d 3^{\prime} G_{\mathrm{HS}}^{(3)}\left[\bar{\nu}+\varphi_{0}\right](1,2,3) G_{\mathrm{HS}}^{(3)}\left[\bar{\nu}+\varphi_{0}\right]\left(1^{\prime}, 2^{\prime}, 3^{\prime}\right) \\
& \times\left\langle\omega(1) \ldots \omega\left(3^{\prime}\right)\right\rangle_{\mathrm{G}}+\mathcal{O}\left(\lambda^{2}\right) \\
& =\lambda \int d 1 \ldots d 3^{\prime} G_{\mathrm{HS}}^{(3)}\left[\bar{\nu}+\varphi_{0}\right](1,2,3) G_{\mathrm{HS}}^{(3)}\left[\bar{\nu}+\varphi_{0}\right]\left(1^{\prime}, 2^{\prime}, 3^{\prime}\right) \\
& \times\left\{\frac{1}{4} \Delta_{\varphi_{0}}(1,2) \Delta_{\varphi_{0}}\left(1^{\prime}, 2^{\prime}\right) \Delta_{\varphi_{0}}\left(3,3^{\prime}\right)\right. \\
& \left.+\frac{1}{6} \Delta_{\varphi_{0}}\left(1,1^{\prime}\right) \Delta_{\varphi_{0}}\left(2,2^{\prime}\right) \Delta_{\varphi_{0}}\left(3,3^{\prime}\right)\right\}+\mathcal{O}\left(\lambda^{2}\right)
\end{aligned}
$$

where once again Wick's theorem (75) was used. Gathering the intermediate results (76) and (77) one has finally, after inspection

$$
\lambda\left\{\langle\delta \mathcal{H}\rangle_{\mathrm{G}}+\frac{1}{2}\left(\left\langle\delta \mathcal{H}^{2}\right\rangle_{\mathrm{G}}-\langle\delta \mathcal{H}\rangle_{\mathrm{G}}^{2}\right)\right\}=\lambda^{2} \ln \bar{\Xi}^{(2)}[\nu]+\mathcal{O}\left(\lambda^{3}\right)
$$

with

$$
\Xi^{(2)}[\nu]=\Xi^{(2)}[\nu] .
$$

We have thus shown that the one and two-loop expressions for $\ln \Xi[\nu]$ coincide in the KSSHE and CV representations. This coincidence is likely to be true at all orders in the loop-expansion.

\section{The pressure and the free energy of the homogeneous fluid}

\subsection{The pressure}

In this section we restrict ourselves to the homogeneous case, therefore $\ln \Xi[\nu]=$ $V \beta P(\nu)$, where $P$ denotes the pressure and $\beta \mathcal{A}[\rho]=V \beta f(\rho)$ where $f$ is the Helmoltz free energy per unit volume. The two-loop expression that we derived for $P$ in Sec. 5 is too much complicated to be of any use in practical calculations since it involves the 3 and 4 density correlation functions of the HS fluid which are unknown, whereas $G_{\mathrm{HS}}^{(2), T}$ is known approximatively, for instance in the Percus-Yevick (PY) approximation [12]. A simple but coherent approximations for $G_{\mathrm{HS}}^{(3), T}$ and $G_{\mathrm{HS}}^{(4), T}$ will be proposed now.

Recall first that it follows from their definitions [26,27] (see e.g. eqs (15)) that the $G_{\mathrm{HS}}^{(n), T}[\nu]$ satisfy to the following relations

$$
\frac{\delta}{\delta \nu(n+1)} G_{\mathrm{HS}}^{(n), T}[\nu](1, \ldots, n)=G_{\mathrm{HS}}^{(n+1), T}[\nu](1, \ldots, n, n+1) .
$$

For a homogeneous system (in which case $\nu$ is a constant) one infers from this 
equation that

$$
\int_{\Omega} d 1 \ldots d n G_{\mathrm{HS}}^{(n+1), T}[\nu](1, \ldots, n, n+1)=\frac{\partial^{n}}{\partial \nu^{n}} \rho_{\mathrm{HS}}(\nu) \equiv \rho_{\mathrm{HS}}^{(n)}(\nu)
$$

where $\rho_{\mathrm{HS}}(\nu)$ is the number density of hard spheres at the chemical potential $\nu$.

In the rest of the section we will adopt the following approximation

$$
G_{\mathrm{HS}}^{(n), T}[\nu](1, \ldots, n)=\rho_{\mathrm{HS}}^{(n+1)}(\nu) \delta(n, 1) \ldots \delta(2,1) \text { for } \mathrm{n} \geq 3 .
$$

Note that this hypothesis is coherent with the exact relations (80) and (81). However, for $n=2$, we adopt for $G_{\mathrm{HS}}^{(2), T}[\nu](1,2)$ some known approximation of the theory of liquids (PY approximation for instance). The free propagator has then a non trivial expression. In Fourier space it reads

$$
\widetilde{\Delta}_{\varphi_{0}}(q)=\frac{\widetilde{w}(q)}{1-\widetilde{w}(q) \widetilde{G}_{\mathrm{HS}}^{(2), T}\left[\bar{\nu}+\varphi_{0}\right](q)}
$$

where

$$
\widetilde{G}_{\mathrm{HS}}^{(2), T}(q)=\int d^{3} x e^{-i q x} G_{\mathrm{HS}}^{(2), T}(x)
$$

is the Fourier transform of $G_{\mathrm{HS}}^{(2), T}[\nu](x)$. The set of approximations that we have just discussed is reasonable as long as the range of the KSSHE field correlation functions is (much) larger than than the range of the HS density correlation functions. This would be true if $w$ is the long range pair interaction or, in general, in the vicinity of the critical point.

With the hypothesis (82) it is easy to obtain the two-loop order approximation for the pressure. One finds

$$
\begin{aligned}
\beta P(\nu) & =\beta P^{(0)}(\nu)+\lambda \beta P^{(1)}(\nu)+\lambda^{2} \beta P^{(2)}(\nu)+\mathcal{O}\left(\lambda^{3}\right), \\
\beta P^{(0)}(\nu) & =\beta P_{\mathrm{MF}}(\nu)=P_{\mathrm{HS}}\left(\bar{\nu}+\varphi_{0}\right)-\frac{\varphi_{0}^{2}}{2 \widetilde{w}(0)}, \\
\beta P^{(1)}(\nu) & =-\frac{1}{2} \int_{\mathbf{q}} \ln \left(1-\widetilde{w}(q) \widetilde{G}_{\mathrm{HS}}^{(2), T}\left[\bar{\nu}+\varphi_{0}\right](q)\right), \\
\beta P^{(2)}(\nu) & =\frac{\rho_{0}^{(3)}}{8} \Delta_{\varphi_{0}}^{2}(0)+\frac{\left[\rho_{0}^{(2)}\right]^{2}}{8} \widetilde{\Delta}_{\varphi_{0}}(0) \Delta_{\varphi_{0}}^{2}(0) \\
& +\frac{\left[\rho_{0}^{(2)}\right]^{2}}{12} \int d^{3} x \Delta_{\varphi_{0}}^{3}(x)
\end{aligned}
$$

where $\rho_{0}^{(n)} \equiv \rho_{\mathrm{HS}}^{(n)}\left(\bar{\nu}+\varphi_{0}\right)$ and $\int_{\mathbf{q}} \equiv \int d^{3} q /(2 \pi)^{3}$. 


\subsection{The free energy}

In order to compute the free energy $f(\rho)$ at the two-loop order we need the expression of the density only at the one-loop order (this a well known properties of Legendre transform [2] that will be checked explicitely further on). We thus define

$$
\begin{aligned}
\rho(\nu) & =\frac{\partial}{\partial \nu} \beta P=\rho_{0}+\Delta \rho(\nu) \\
\Delta \rho(\nu) & =\lambda \rho^{(1)}(\nu)+\mathcal{O}\left(\lambda^{2}\right),
\end{aligned}
$$

where $\rho_{0} \equiv \rho_{\mathrm{HS}}\left(\bar{\nu}+\varphi_{0}\right)$ and

$$
\begin{aligned}
\rho^{(1)}(\nu) & =\frac{\partial}{\partial \nu} \beta P^{(1)}(\nu)=\frac{1}{2} \int_{\mathbf{q}} \frac{\widetilde{w}(q)}{1-\widetilde{w}(q) \widetilde{G}_{\mathrm{HS}}^{(2), T}(q)} \frac{\partial}{\partial \nu} \widetilde{G}_{\mathrm{HS}}^{(2), T}(q) \\
& =\frac{\rho_{0}^{(2)}}{2} \Delta_{\varphi_{0}}(0)\left(1+\frac{\partial \varphi_{0}}{\partial \nu}\right),
\end{aligned}
$$

where, in order to derive the last line, we made use of eqs. (80) and (82). In order to obtain the expression of $\partial \varphi_{0} / \partial \nu$ one derives the homogeneous stationary condition (38) with respect to $\nu$ which gives us

$$
1+\frac{\partial \varphi_{0}}{\partial \nu}=\frac{1}{1-\rho_{0}^{(1)} \widetilde{w}(0)} .
$$

Introducing this result in the last line of eq. (87) we find finally that

$$
\rho^{(1)}(\nu)=\frac{\rho_{0}^{(2)}}{2} \frac{\Delta_{\varphi_{0}}(0)}{1-\rho_{0}^{(1)} \widetilde{w}(0)} .
$$

We are now in position to compute the free energy

$$
\begin{aligned}
\beta f(\rho) & =\rho \nu-\beta P(\nu) \\
& =\rho \nu-\beta P^{(0)}(\nu)-\lambda \beta P^{(1)}(\nu)-\lambda^{2} \beta P^{(2)}(\nu)+\mathcal{O}\left(\lambda^{3}\right) .
\end{aligned}
$$

Our task is now to reexpress the r.h.s. as a function of $\rho=\rho_{0}+\Delta \rho(\nu)$ which will be done along the same lines that those used in [18]. We shall compute separately three contributions to $\beta f$.

i) Computation of $X \equiv \rho \nu-\beta P^{(0)}(\nu)$

We first note that $X$ can be rewritten as

$$
X=\rho_{0} \nu-\beta P^{(0)}(\nu)+\nu \Delta \rho=\beta f^{(0)}\left(\rho_{0}\right)+\nu \Delta \rho,
$$


where $f^{(0)}\left(\rho_{0}\right) \equiv f_{\mathrm{MF}}\left(\rho_{0}\right)$ is the MF free energy of the homogeneous system at the density $\rho_{0}$, i.e. (cf. eq. (42))

$$
\beta f^{(0)}\left(\rho_{0}\right)=\beta f_{\mathrm{HS}}\left(\rho_{0}\right)-\frac{1}{2} \rho_{0} \widetilde{w}^{2}(0)+\frac{1}{2} \rho_{0} w(0) .
$$

In order to relate $f^{(0)}\left(\rho_{0}\right)$ to $f(\rho]$ we perform a second order Taylor expansion

$$
\beta f^{(0)}\left(\rho=\rho_{0}+\Delta \rho\right)=\beta f^{(0)}\left(\rho_{0}\right)+\nu \Delta \rho-\frac{1}{2} \widetilde{C}_{M F}(0)[\Delta \rho]^{2}+\mathcal{O}\left(\lambda^{3}\right),
$$

where the Fourier transform at $q=0$ of the MF two-body vertex function is computed from eq. (44b) with the result

$$
\widetilde{C}_{M F}(0)=-1 / \widetilde{G}_{H S}(0)+\widetilde{w}(0)=-\frac{1-\rho_{0}^{(1)} \widetilde{w}(0)}{\rho_{0}^{(1)}} .
$$

Combining the intermediate results (92), (93), and (94) one has finally

$$
\begin{aligned}
X & =\beta f^{(0)}(\rho)+\frac{1}{2} \widetilde{C}_{M F}(0)[\Delta \rho]^{2}+\mathcal{O}\left(\lambda^{3}\right) \\
& =\beta f^{(0)}(\rho)-\frac{\lambda^{2}}{8} \Delta_{\varphi_{0}}^{2}(0) \frac{\left[\rho_{0}^{(2)}\right]^{2}}{\rho_{0}^{(1)}\left(1-\rho_{0}^{(1)} \widetilde{w}(0)\right)}+\mathcal{O}\left(\lambda^{3}\right) .
\end{aligned}
$$

We note that, as claimed at the beginning of the paragraph, the knowledge of $\Delta \rho$ at the one-loop order is sufficient for our purpose.

ii) Computation of $\beta P^{(1)}(\nu)$

As can be inferred from eq. (85) $\beta P^{(1)}(\nu)$ may be seen as a function of the sole variable $\rho_{0} \equiv \rho_{H S}\left(\bar{\nu}+\varphi_{0}\right)$ since for the HS reference system there is a one to one correspondence between densities and chemical potentials -at least in the fluid phase, away from the liquid/solid transition-. Therefore $\beta P^{(1)}(\nu) \equiv \beta P^{(1)}\left(\rho_{0}\right)$, with some abusive notations. One thus have

$$
\begin{aligned}
\beta P^{(1)}\left(\rho=\rho_{0}+\Delta \rho\right) & =\beta P^{(1)}\left(\rho_{0}\right)+\frac{\partial \nu}{\partial \rho_{0}} \frac{\partial \beta P^{(1)}(\nu)}{\partial \nu} \Delta \rho+\mathcal{O}(\lambda), \\
& =\beta P^{(1)}\left(\rho_{0}\right)+\frac{\partial \nu}{\partial \rho_{0}} \frac{[\Delta \rho]^{2}}{\lambda}+\mathcal{O}(\lambda),
\end{aligned}
$$

where the last line follows from eqs. (86) and (87). At this point we notice that

$$
\frac{\partial \rho_{0}}{\partial \nu}=\widetilde{G}_{\mathrm{MF}}^{T}(q=0)=\frac{\rho_{0}^{(1)}}{1-\rho_{0}^{(1)} \widetilde{w}(0)}=1 / \frac{\partial \nu}{\partial \rho_{0}},
$$


which allows us to write finally

$$
\begin{aligned}
& \beta P^{(1)}\left(\rho_{0}\right)=\beta P^{(1)}(\rho)-\frac{\lambda}{4} \Delta_{\varphi_{0}}^{2}(0) \frac{\left[\rho_{0}^{(2)}\right]^{2}}{\rho_{0}^{(1)}\left(1-\rho_{0}^{(1)} \widetilde{w}(0)\right)} \\
& \beta P^{(1)}(\rho)=-\frac{1}{2} \int_{q} \ln \left(1-\widetilde{w}(q) \widetilde{G}_{\mathrm{HS}, \rho}^{T}(q)\right),
\end{aligned}
$$

where, in the second equality, the subscript emphasizes that the truncated HS pair correlation function has to be computed at the density $\rho$.

The computation of $f(\rho)$ is now completed and gathering the intermediate results one has finally, setting $\lambda=1$

$$
\begin{aligned}
\beta f(\rho) & =\beta f_{\mathrm{HS}}(\rho)-\frac{\widetilde{w}(0)}{2} \rho^{2} \\
& +\frac{1}{2} \int_{\mathbf{q}}\left\{\ln \left(1-\widetilde{w}(q) \widetilde{G}_{\mathrm{HS}, \rho}^{T}(q)\right)+\rho \widetilde{w}(q)\right\} \\
& -\frac{\rho_{0}^{(3)}}{8} \Delta_{\rho}^{2}(0)+\frac{1}{8} \Delta_{\rho}^{2}(0) \frac{\left[\rho_{0}^{(2)}\right]^{2}}{\rho_{0}^{(1)}}-\frac{\left[\rho_{0}^{(2)}\right]^{2}}{12} \int d^{3} x \Delta_{\rho}^{3}(x) .
\end{aligned}
$$

Some comments on this result are in order.

i) The last line in eq. (99) gathers the two-loop contributions of $\beta f(\rho)$. At this order of the loop expansion it is thus legitimate to replace $\Delta_{\varphi_{0}}$ by $\Delta_{\rho}$ where the propagator is evaluated at the density $\rho$ rather than $\rho_{0}$ since $\rho-\rho_{0}=\mathcal{O}(\lambda)$. More explicitly

$$
\widetilde{\Delta}_{\rho}(q)=\frac{\widetilde{w}(q)}{1-\widetilde{G}_{\mathrm{HS}, \rho}^{T}(q) \widetilde{w}(q)}
$$

ii) Similarly the derivatives $\rho_{0}^{(n)}$ which enter the last line of eq. (99) can be evaluated at the density $\rho$ rather than $\rho_{0}$. One can thus write, taking advantage of the one to one correspondence between the HS densities and chemical potentials

$$
\begin{aligned}
& \rho_{0}^{(1)}=\frac{1}{\nu_{\mathrm{HS}}^{(1)}(\rho)} \\
& \rho_{0}^{(2)}=\frac{\partial \rho_{0}^{(1)}}{\partial \nu}=\frac{-\nu_{\mathrm{HS}}^{(2)}(\rho)}{\left[\nu_{\mathrm{HS}}^{(1)}(\rho)\right]^{3}} \\
& \rho_{0}^{(3)}=\frac{\partial \rho_{0}^{(2)}}{\partial \nu}=\frac{3\left[\nu_{\mathrm{HS}}^{(2)}(\rho)\right]^{2}-\nu_{\mathrm{HS}}^{(3)}(\rho) \nu_{\mathrm{HS}}^{(1)}(\rho)}{\left[\nu_{\mathrm{HS}}^{(1)}(\rho)\right]^{5}},
\end{aligned}
$$


where $\nu_{\mathrm{HS}}^{(n)}(\rho)$ denotes the $n$th derivative of the HS chemical potential with respect to the density (it can be computed in the framework of the PY or Carnahan-Starling approximations for instance [12]).

iii) It must be pointed out that, quite unexpectedly, the reducible diagram $D_{b}$ has not be cancelled by the Legendre transform. Usually, in statistical field theory it is the case (cf. [2]). The reason is that the chemical potential $\nu$ is not the field conjugate to the order parameter $m=\langle\varphi\rangle_{K}$ of the KSSHE field theory. However one of us have shown elsewhere $[18,23]$ that for the symmetric mixtures of charged hard spheres only irreducible diagrams contribute to $\beta f^{(2)}$.

iv) All the quantities which enter eq. (99) can be computed numerically (for instance in the PY approximation). We plan to test the validity of our approximation in future work.

\section{Conclusion}

Using the CV method we reconsider the basic relations of statistical field theory of simple fluids that follow from this approach. In contrary to the KSSHE theory [11] the corresponding CV action depends on two scalar fields - field $\rho$ connected to the number density of particles and field $\omega$ conjugate to $\rho$. Explicit expressions that allow to relate between themselves the correlation functions defined in different versions of the theory are derived.

For a one-component continuous model of fluids, consisting of hard spheres interacting through the short-range pair potential, we calculated the grand partition function in a systematic way for both versions of statistical field theory using the loop expansion technique. As it was expected, in all the orders of loop expansion considered, both versions of the theory produced indeed the same analytical results. The expressions for the pressure as well as for the free energy are derived in a two-loop approximation that is the next step in comparison with the results obtained recently by one of us [11] within the KSSHE theory. In fact this is a new type of approximation which we plan to test in our future work.

In contrast to the usual statistical field theory [2], our results demonstrate that for the case of simple fluids the reducible diagram is not cancelled by the Legendre transform. It is due to the fact that our field theory is a nonstandard in the sense that the coupling between the internal field $\varphi$ and the external field $\nu$ is non-linear yielding new expressions for $\beta f(\rho)$.

From our analysis of the CV and KSSHE transformations we can also conclude that the former has some important advantages which could be very 
useful for more complicate models of fluids. In particular, it is valid for an arbitrary pair potential (including a pair interaction $w(1,2)$ which does not possess an inverse) and is easily generalized for the case of n-body interparticle interactions with $n>2$.

\section{Acknowledgments}

This work was made in the framework of the cooperation project between the CNRS and the NASU (ref. CNRS 17110).

\section{A Functional measures and integrals}

In this appendix we give some details on functional measures and integrals. Let us consider a real scalar field $\varphi(\mathbf{x})$ defined in a cube $\mathcal{C}_{3}$ of side $L$ and volume $V=L^{3}$. We assume periodic boundary conditions, i.e. we restrict ourselves to fields which can be written as a Fourier series,

$$
\varphi(\mathbf{x})=\frac{1}{L^{3}} \sum_{\mathbf{q} \in \Lambda} \widetilde{\varphi}_{\mathbf{q}} e^{i \mathbf{q} \mathbf{x}}
$$

where $\Lambda=(2 \pi / L) \mathbf{Z}^{3}$ is the reciprocal cubic lattice. The reality of $\varphi \mathrm{im}-$

plies that, for $\mathbf{q} \neq 0 \widetilde{\varphi}_{-\mathbf{q}}=\widetilde{\varphi}_{\mathbf{q}}^{\star}$, where the star means complex conjugation. Following Wegner [19] we define the normalized functional measure $\mathcal{D} \varphi$ as

$$
\begin{aligned}
\mathcal{D} \varphi & \equiv \prod_{\mathbf{q} \in \Lambda} \frac{d \widetilde{\varphi}_{\mathbf{q}}}{\sqrt{2 \pi V}} \\
d \widetilde{\varphi}_{\mathbf{q}} d \widetilde{\varphi}_{-\mathbf{q}} & =2 d \Re \widetilde{\varphi}_{\mathbf{q}} d \Im \widetilde{\varphi}_{\mathbf{q}} \text { for } \mathbf{q} \neq 0 .
\end{aligned}
$$

Eq. (A.2) can be conveniently rewritten as

$$
\mathcal{D} \varphi=\frac{d \varphi_{0}}{\sqrt{2 \pi V}} \prod_{\mathbf{q} \in \Lambda^{\star}} \frac{d \Re \widetilde{\varphi}_{\mathbf{q}} d \Im \widetilde{\varphi}_{\mathbf{q}}}{\pi V}
$$

where the sum in the r.h.s runs over only the half $\Lambda^{*}$ of all the vectors of the reciprocal lattice $\Lambda$ (for instance those with $q_{x} \geq 0$ ). With the definition (A.2) we have 


$$
\begin{aligned}
\mathcal{N}_{w} & =\int \mathcal{D} \varphi \exp \left(-\frac{1}{2}\left\langle\varphi\left|w^{-1}\right| \varphi\right\rangle\right) \\
& =\exp \left(\frac{1}{2} \sum_{\mathbf{q} \in \Lambda} \ln \widetilde{w}(q)\right) \\
\stackrel{L \rightarrow \infty}{\longrightarrow} & \exp \left(\frac{V}{2} \int_{\mathbf{q}} \ln \widetilde{w}(q)\right),
\end{aligned}
$$

where $w(1,2)$ is positive and satisfies $w(1,2)=w(2,1) \equiv w\left(x_{12}\right)$. It is worth noting that in this case we have trivially $\mathcal{N}_{w^{-1}}=1 / \mathcal{N}_{w}$. More generally and with the same hypothesis we have the useful identity

$$
\begin{aligned}
\langle\exp ((i)\langle\varphi \mid \omega\rangle)\rangle_{w} & \equiv \mathcal{N}_{w}^{-1} \int \mathcal{D} \varphi \exp \left(-\frac{1}{2}\left\langle\varphi\left|w^{-1}\right| \varphi\right\rangle+(i)\langle\varphi \mid \omega\rangle\right) \\
& =\exp \left(+(-) \frac{1}{2}\langle\omega|w| \omega\rangle\right)
\end{aligned}
$$

where $\omega$ is a real scalar field.

We define now the "functional delta" distribution $\delta_{\mathcal{F}}[\lambda]$ as $[1]$

$$
\delta_{\mathcal{F}}[\lambda] \equiv \int \mathcal{D} \omega \exp (i\langle\omega \mid \lambda\rangle)
$$

where both $\omega$ and $\lambda$ are real scalar fields defined on $\mathcal{C}$. Since

$$
\langle\omega \mid \lambda\rangle=\int_{\mathcal{C}} d^{3} \mathbf{x} \omega(\mathbf{x}) \lambda(\mathbf{x})=\frac{1}{V} \sum_{\mathbf{q} \in \Lambda} \widetilde{\omega}_{-\mathbf{q}} \widetilde{\lambda}_{\mathbf{q}},
$$

it follows from eq. (A.2) that we have more explicitly

$$
\delta_{\mathcal{F}}[\lambda]=\sqrt{2 \pi V} \delta\left(\widetilde{\lambda}_{0}\right) \prod_{\mathbf{q} \in \Lambda^{\star}}\left[\pi V \delta\left(\Re \widetilde{\lambda}_{\mathbf{q}}\right) \delta\left(\Im \widetilde{\lambda}_{\mathbf{q}}\right)\right]
$$

Therefore

$$
\int \mathcal{D} \lambda \delta_{\mathcal{F}}[\lambda]=1
$$

and, more generally

$$
\int \mathcal{D} \lambda \mathcal{F}[\lambda] \delta_{\mathcal{F}}\left[\lambda-\lambda_{0}\right]=\mathcal{F}\left[\lambda_{0}\right]
$$

where $\mathcal{F}[\lambda]$ is some arbitrary functional of the field $\lambda(x)$.

\section{B Two useful identities}

We prove here two identities used in Sec. 5. Firstly let $K(\mathbf{x}, \mathbf{y})$ be a definite positive operator assumed to be symmetric. The variables $\mathbf{x}$ and $\mathbf{y}$ take their 
values in the cube $\mathcal{C}_{3}$ with periodic boundary conditions. We define (provided that it exists)

$$
\mathcal{N}_{K}=\int \mathcal{D} \varphi \exp \left(-\frac{1}{2}\left\langle\varphi\left|K^{-1}\right| \varphi\right\rangle\right)
$$

and we want to prove that $\mathcal{N}_{K^{-1}}=1 / \mathcal{N}_{K}$. If $K(\mathbf{x}, \mathbf{y})=K(\mathbf{x}-\mathbf{y})=K(\mathbf{y}-\mathbf{x})$, i.e. in the homogeneous case, it is trivial as pointed out in Appendix A. In the general case we still have [2]

$$
\int \mathcal{D} \varphi \exp \left(-\frac{1}{2}\left\langle\varphi\left|K^{-1}\right| \varphi\right\rangle+i\langle J \mid \varphi\rangle\right)=\mathcal{N}_{K} \exp \left(-\frac{1}{2}\langle J|K| J\rangle\right)
$$

where $J$ is some real scalar field. Therefore

$$
\begin{aligned}
\mathcal{N}_{K^{-1}} & =\int \mathcal{D} J \exp \left(-\frac{1}{2}\langle J|K| J\rangle\right) \\
& =\frac{1}{\mathcal{N}_{K}} \int \mathcal{D} J \mathcal{D} \varphi \exp \left(-\frac{1}{2}\left\langle\varphi\left|K^{-1}\right| \varphi\right\rangle+i\langle J \mid \varphi\rangle\right) \\
& =\frac{1}{\mathcal{N}_{K}} \int \mathcal{D} \varphi \exp \left(-\frac{1}{2}\left\langle\varphi\left|K^{-1}\right| \varphi\right\rangle\right) \delta_{\mathcal{F}}[\varphi] \\
& =\frac{1}{\mathcal{N}_{K}}
\end{aligned}
$$

where we have made use of the representation (A.6) of $\delta_{\mathcal{F}}[\varphi]$.

Secondly let us consider two definite positive symmetric operators $a(\mathbf{x}, \mathbf{y})$ and $b(\mathbf{x}, \mathbf{y})$. We define (provided that these objects exist)

$$
\left\langle\exp \left(\frac{1}{2}\langle\varphi|a| \varphi\rangle\right)\right\rangle_{b}=\frac{\int \mathcal{D} \varphi \exp \left(-\frac{1}{2}\left\langle\varphi\left|b^{-1}\right| \varphi\right\rangle+\frac{1}{2}\langle\varphi|a| \varphi\rangle\right)}{\int \mathcal{D} \varphi \exp \left(-\frac{1}{2}\left\langle\varphi\left|b^{-1}\right| \varphi\right\rangle\right)}
$$

and we want to prove that

$$
\left\langle\exp \left(\frac{1}{2}\langle\varphi|a| \varphi\rangle\right)\right\rangle_{b}=\left\langle\exp \left(\frac{1}{2}\langle\varphi|b| \varphi\rangle\right)\right\rangle_{a}
$$

Note that these expressions are well defined only if $\left(a-b^{-1}\right)>0$ and $\left(b-a^{-1}\right)>$ 0 , i.e. $a b<1$. If $a(b)(\mathbf{x}, \mathbf{y})=a(b)(\mathbf{x}-\mathbf{y})=a(b)(\mathbf{y}-\mathbf{x})$ (i.e. in the homogeneous case) the result is a trivial consequence of eq. (A.5).

In the general case one proceeds as follows. Since $a>0$ and $b>0$ then one can define the operators $a^{ \pm 1 / 2}$ and $b^{ \pm 1 / 2}$ which are all symmetric and positive definite. Let us perform the change of variable

$$
\varphi(1)=b^{1 / 2}\left(1,1^{\prime}\right) a^{-1 / 2}\left(1^{\prime}, 1^{\prime \prime}\right) \psi\left(1^{\prime \prime}\right)
$$

in eq. (B.4). The functional Jacobian of the transform $J=\operatorname{det} \delta \varphi(1) / \delta \psi(2)=$ $\operatorname{det} b^{1 / 2}\left(1,1^{\prime}\right) a^{-1 / 2}\left(1^{\prime}, 2\right)$ is a non zero constant which can be picked out of the 
integral. Moreover it is easy to show that

$$
\left\langle\varphi\left|b^{-1}\right| \varphi\right\rangle=\left\langle\psi\left|a^{-1}\right| \psi\right\rangle, \quad\langle\varphi|a| \varphi\rangle=\langle\psi|b| \psi\rangle
$$

therefore one has

$$
\begin{aligned}
\left\langle\exp \left(\frac{1}{2}\langle\varphi|a| \varphi\rangle\right)\right\rangle_{b} & =\frac{J \int \mathcal{D} \psi \exp \left(-\frac{1}{2}\left\langle\psi\left|a^{-1}\right| \psi\right\rangle+\frac{1}{2}\langle\psi|b| \psi\rangle\right)}{J \int \mathcal{D} \psi \exp \left(-\frac{1}{2}\left\langle\psi\left|a^{-1}\right| \psi\right\rangle\right)} \\
& =\left\langle\exp \left(\frac{1}{2}\langle\varphi|b| \varphi\rangle\right)\right\rangle_{a} .
\end{aligned}
$$

\section{References}

[1] Negele J. W.and Orland H., Quantum Many-Particle Systems, (Frontiers in Physics, Addison-Wesley, 1988).

[2] Zinn-Justin J., Quantum Field Theory and Critical Phenomena, (Clarendon Press, Oxford, 1989).

[3] J. Hubbard, Phys. Rev. Lett. 3, 77 (1959).

[4] R. L. Stratonovich, Sov. Phys. Solid State 2, 1824 (1958).

[5] D.N. Zubarev, Dokl. Akad. Nauk SSSR 95, 757 (1954) [in Russian].

[6] I. R. Yukhnovskii, Zh. Eksp. Teor. Fiz. 34, 379 (1958) [Sov. Phys.JETP 34, 263 (1958)].

[7] D. Bohm and D. Pines, Phys. Rev. 82, 625 (1951).

[8] Yukhnovskii I. R. and Holovko M.F.Statistical Theory of Classical Equilibrium Systems,(Naukova Dumka, Kiev, 1980) [in Russian].

[9] Yukhnovskii I. R., Phase Transitions of the Second Order, Collective variable methods, (World Scientific, Singapore, 1987).

[10] A. Ciach, G. Stell, J. Mol. Liq. 87, 253 (2000).

[11] J.-M. Caillol, Mol. Phys. 101, 1617 (2003).

[12] Hansen J.-P. and Mc Donald I. R., Theory of Simple Liquids (Academic Press, London, 1986).

[13] M. Kac, Phys. Fluids 2, 8 (1959).

[14] A. J. F. Siegert, Physica 26, 530 (1960).

[15] J. Hubbard and P. Schofield, Phys. Lett. 40A, 245 (1972).

[16] S. F. Edwards, Phil. Mag. 4, 1171 (1959). 
[17] F. W. Wiegel, Phys. Rep. 16C, 57 (1975).

[18] J.-M. Caillol, J. Stat. Phys. 115, 1461 (2004); extended version: arXiv:cond-mat/0305465

[19] Wegner F. J., in Phase Transitions and Critical Phenomena Vol. VI, C. Domb and M. S. Green, eds (Academic Press, New York, 1976).

[20] Ruelle D., Statistical Mechanics, Rigorous Results, (New York, W. A. Benjamin, New York, 1969).

[21] G. V. Efimov and E. A. Nogovitsin, Physica A 234, 506 (1996).

[22] J.-M. Caillol, J. Phys. A: Math. Gen. 36, 10431 (2003).

[23] J.-M. Caillol, Mol. Phys. 103, 1271 (2005).

[24] I. R. Yukhnovskii, Physica A 168, 999 (1990).

[25] I. R. Yukhnovskii and O. V. Patsahan, J. Stat. Phys. 81, 647 (1995).

[26] Stell G. , The Equilibrium Theory of Classical Fluids, ed H. L. Frisch and J. L. Lebowitz, (Benjamin, New York, 1964).

[27] Stell G., Phase Transitions and Critical Phenomena, Vol 5b, ed. C. Domb and J. L. Lebowitz, (Academic Press, 1973). 